\title{
FAILURE OF A QUADRATIC ANALOGUE OF SERRE'S CONJECTURE
}

\author{
BY S. PARIMALA \\ Communicated by Olga Taussky Todd, July 30, 1976
}

Let $A$ be a commutative ring with identity. By an inner product $A$-space we shall understand, as in [6], a pair $(P, q)$, where $P$ is a finitely generated projective $A$-module and $q$ is a symmetric bilinear form $P \times P \rightarrow A$ which is nonsingular (i.e. induces an isomorphism $P \stackrel{\sim}{\longrightarrow} P^{*}$ ). If $B$ is a commutative $A$-algebra we obtain an inner product $B$-space $\left(B \otimes_{A} P, B \otimes_{A} q\right)$. Inner product $B$-spaces isomorphic to one of these will be said to be extended from $A$.

The quadratic analogue of Serre's conjecture is the affirmation of:

Suppose $A$ is a polynomial algebra $K\left[X_{1}, \ldots, X_{n}\right]$ over a field $K$.

Is every inner product $A$-space extended from $K$ ?

This question is motivated by the following evidence.

(1) Serre's conjecture that projective $A$-modules are free, hence extended from $K$, has recently been proved by Quillen and Suslin (cf. [4]). Moreover this immediately implies that "symplectic $A$-spaces" are extended from $K$ (see e.g. [1, Chapter IV, (4.11.2)]).

(2) If $\operatorname{Char}(K) \neq 2$ then a theorem of Karoubi [7, Theorem 1.1] implies that every inner product $A$-space is stably isomorphic to one extended from $K$.

(3) A theorem of Harder (see [8, Theorem 13.4.3]) gives an affirmative response to (QS) for $n=1$.

A major tool in Quillen's proof of Serre's conjecture is:

QUILlEN'S LOCALIZATION THEOREM [11]. Let $A$ be a commutative ring, let $T$ be an indeterminate, and let $M$ be a finitely presented $A[T]$-module. If, for all maximal ideals m of $A, M_{\mathrm{m}}$ is extended from $A_{\mathrm{m}}$, then $M$ is extended from $A$.

(4) The analogue of Quillen's localization theorem for inner product spaces has been proved in [3].

The other main tool Quillen uses is:

HorRock's THEOREM [5]. Let $A$ be a local ring and let $P$ be a finitely generated projective $A[T]$-module. If $P$ extends to a locally free sheaf on $\mathbf{P}_{A}^{1}$, then $P$ is extended from $A$ (hence free).

AMS (MOS) subject classifications (1970). Primary 15A63; Secondary 18F25. 
It is natural then to ask:

(QH) Is the analogue of Horrock's theorem for inner product spaces valid?

We here answer (QS) and $(\mathrm{QH})$ negatively, with the following example. Let $A=\mathbf{R}[X, Y]$. Consider the symmetric $4 \times 4$ matrix over

$$
A, S=\left(\begin{array}{cc}
\alpha & \beta \\
t^{\beta} & \alpha
\end{array}\right)
$$

where

$$
\alpha=\left(\begin{array}{cc}
4+Y^{2}\left(1+X^{2}\right) & X Y\left(1+Y^{2}\right) \\
X Y\left(1+Y^{2}\right) & 1+X^{2} Y^{4}
\end{array}\right)={ }^{t} \alpha,
$$

( $t$ denotes transpose), and

$$
\beta=\left(\begin{array}{cc}
0 & Y\left(1+X^{2} Y^{2}\right) \\
-Y\left(1+X^{2} Y^{2}\right) & 0
\end{array}\right)=-{ }^{t} \beta
$$

Let $q$ be the bilinear form on $P=A^{4}$ with matrix $S$ relative to the natural basis of $A^{4}$.

THEOREM. (1) $(P, q)$ is an inner product space over $A=\mathbf{R}[X, Y]$ which is not extended from $\mathbf{R}$.

(2) For each prime ideal $p$ of $A$, the inner product $A_{p}$-space $\left(P_{p}, q_{p}\right)$ is extended from $\mathbf{R}$.

(3) $(P, q)$ extends to a sheaf of inner product spaces over $\mathbf{P}_{\mathbf{R}[X]}^{1}$, yet for some prime ideal fo of $\mathbf{R}[X]$, the inner product $\mathbf{R}[X]_{p}[Y]$-space $\left(P_{p}, q_{p}\right)$ is not extended from $\mathbf{R}[X]_{p}$.

REMARKS. (a). The matrix $S$ is derived from the hermitian matrix $H=$ $\alpha+i \beta$ over $\mathbf{C}[X, Y]$, which was discovered from an investigation of the classification of the rank 1 projective $\mathbf{H}[X, Y]$-modules $(\mathbf{H}=$ quaternions) in terms of hermitian matrices, established in [9], [10]. The analogue of the above theorem for the hermitian $\mathbf{C}[X, Y]$-space defined by $H$ is also valid.

(b) The matrix $S$ has entries in $\mathrm{Z}[X, Y]$, and $\operatorname{det}(S)=16$. Thus $(P, q)$ is extended from an inner product space over $Z[1 / 2][X, Y]$.

(c) If one considers quadratic forms rather than symmetric bilinear forms the analogue of (QS) has a negative response (in characteristic 2 of course) alalready for $n=1$ (see [7, p. 318]).

(d) Bass [2] has investigated (QS) when $K$ is algebraically closed.

(e) It follows from Harder's theorem (3) that the answer to $(\mathrm{QH})$ is trivially in the affirmative if $\mathrm{A}$ is a field. Our theorem shows that the answer is negative already for the discrete valuation ring $\mathbf{R}[X]_{p}$.

(f) The proof of the above theorem will appear elsewhere. 
I am thankful to Professor H. Bass for carefully going through my calculations and his great help in preparing this manuscript.

I am more than grateful to Professor R. Sridharan to whom this work is respectfully dedicated.

\section{REFERENCES}

1. H. Bass, Unitary algebraic K-theory, Algebraic $K$-theory. III (Proc. Conf., Seattle Res. Center, Battelle Memorial Inst., 1972), Lecture Notes in Math., vol. 343, SpringerVerlag, Berlin and New York, 1973, pp. 57-265. MR 51 \#8211.

2. - Quadratic modules over polynomial rings (to appear).

3. H. Bass, E. H. Connell, and D. L. Wright, Locally polynomial algebras are symmetric algebras (to appear).

4. D. Ferrand, Les modules projectifs de type fini sur un anneau de polynômes sur un corps sont libres, Séminaire Bourbaki, Exposé 484, Juin 1976.

5. G. Horrocks, Projective modules over an extension of a local ring, Proc. London Math. Soc. (3) 14 (1964), 714-718. MR 30 \#121.

6. D. Husemoller and J. Milnor, Symmetric bilinear forms, Ergebnisse der Mathematik, Band 73, Springer-Verlag, Berlin and New York, 1973.

7. M. Karoubi, Périodicité de la K-théorie hermitienne, Algebraic K-theory. III (Proc. Conf. Seattle Res. Center, Battelle Memorial Inst., 1972), Lecture Notes in Math. vol. 343, Springer-Verlag, Berlin and New York, 1973, pp. 301-411. MR 48 \#656c.

8. M. Knebusch, Grothendieck- und Wittringe von nichtausgearteten symmetrischen Bilinearformen, S.-B. Heidelberger Akad. Wiss. Math.-Natur. K1. 1969/70, 93-157. MR 42 \#6001.

9. S. Parimala, Projective modules and hermitian matrices, J. Pure Applied Algebra 7 (1976), 5-14.

10. S. Parimala and R. Sridharan, Projective modules over polynomial rings over division rings, J. Math. Kyoto Univ. 15 (1975), 129-148. MR 5112935.

11. D. Quillen, Projective modules over polynomial rings, Invent. Math. (to appear).

SCHOOL OF MATHEMATICS, TATA INSTITUTE OF FUNDAMENTAL RESEARCH, BOMBAY -5, INDIA 